\title{
Monthly Forecasting of the Dollar to the Ruble Exchange Rate with Adaptive Kalman Filter
}

\author{
Sergei Borodachev \\ Graduate School of Economics and Management, Ural Federal University, Ekaterinburg, Russia \\ Email address: \\ s.m.borodachev@gmail.com
}

\section{To cite this article:}

Sergei Borodachev. Monthly Forecasting of the Dollar to the Ruble Exchange Rate with Adaptive Kalman Filter. International Journal of Systems Science and Applied Mathematics. Vol. 3, No. 2, 2018, pp. 24-29. doi: 10.11648/j.ijssam.20180302.12

Received: June 1, 2018; Accepted: June 19, 2018; Published: July 13, 2018

\begin{abstract}
The goal: to develop a model that allows you to forecast the dollar to the ruble exchange rate for a month ahead based on macroeconomic data, published at monthly intervals. Proposed structural model of the dynamics of the ruble and dollar masses that determine the exchange rate, depending on changes in foreign exchange reserves, the balance of foreign trade, the monetary base, the MICEX index, the price of oil. With the help of the Kalman filter (KF), the model parameters, the dynamics of the money masses were estimated, and forecasting of the dollar exchange rate was done. Monthly data were used from the beginning of 2015 to mid-2017. The estimation of the capacity of dollar market was found in about half the capacity of the MICEX index funds. Average error of forecasts, based on information available one step before the forecasted moments (RMSEA) was 1.99. Adaptive form of KF was developed when, similarly to the EM algorithm, the phases of KF estimation in the window and minimization of average prediction error to determine the optimal estimates for the system model parameters in this moment are sequentially alternated. With this RMSEA became 1.39 .
\end{abstract}

Keywords: Currency Market, Adaptive Kalman Filter, Exchange Rates, Prediction Error of Adequacy

\section{Introduction}

Many studies have been devoted to the forecasting of exchange rates $[1,2]$; in particular, a model of daily forecasting has been proposed in [3]. In this paper, an attempt is made to build monthly forecasts. Particularly, such forecasts are necessary when investing in Dual Currency Deposits. Of course, the changes in the dollar-ruble exchange rate, especially short-term ones, are greatly influenced by information shocks and crises, so a relatively quiet period was chosen for the training of the model and its testing from the end of 2014 to mid-2017. Further in the article $t$ - the moments of the beginning of the months, take the values from 0 to $T-1$ that is from January 1, 2015 to May 1, 2017 (total $T=29$ months).

\section{Model and Data}

Let $R d^{t}$ denote the rubles mass, and $D^{t}$ the corresponding mass of dollars participating in the exchange and determining the proper dollar / ruble rate at time $t$ :

$$
S^{t}=\frac{R d^{t}}{D^{t}}
$$

We will assume that changes in these masses depend on changes in the following macroeconomic variables.

$$
\begin{gathered}
R d^{t+1}=R d^{t}+\beta_{0} \Delta M^{t-1}+\beta_{1} \Delta M^{t}+\beta_{2} \Delta R^{t}, \\
D^{t+1}=D^{t}+\gamma_{0} \Delta B^{t}+\gamma_{1} \Delta C a^{t}+\gamma_{2} \Delta M^{t}+\gamma_{3} \Delta S a^{t},
\end{gathered}
$$

Here all the differences are backward $\Delta O^{t}=O^{t}-O^{t-1}$.

$M^{t}$ - MICEX index, $R^{t}$ - a monetary base in a narrow definition (includes cash issued by the Bank of Russia (including balances in cash departments of credit institutions) and balances on accounts of mandatory reserves for funds borrowed by credit institutions in the national currency deposited with the Bank of Russia) bn., $B^{t}$ - the price of a barrel of oil $\$, \mathrm{Ca}^{t}$ - cash currency and deposits included in the item "International reserve assets and other liquidity in foreign currency of the Russian Federation" \$ trillion, $S a^{t}$ is the monthly balance of the trade balance of the "Foreign 
Trade of the Russian Federation with goods" \$ bn. All these data, as well as the exchange rate $Y^{t}$ - "Nominal rates of foreign currencies to the ruble (rubles per unit of foreign currency)" are available on the Bank of Russia website http: / /www.cbr.ru. See Table 1.

Table 1. Dollar-ruble exchange rate and macroeconomic variables affecting it.

\begin{tabular}{|c|c|c|c|c|c|c|c|c|c|c|}
\hline $\mathbf{t}$ & $\mathbf{0}$ & 1 & 2 & 3 & $\ldots$ & 24 & 25 & 26 & 27 & 28 \\
\hline$Y^{t}$ & 59.23 & 69.98 & 61.52 & 58.08 & $\ldots$ & 61.3982 & 60.1142 & 58.3132 & 56.235 & 56.98 \\
\hline$M^{t}$ & 1396.61 & 1647.69 & 1758.97 & 1626.18 & $\ldots$ & 2232.72 & 2217.39 & 2035.77 & 1995.9 & 2016.71 \\
\hline$R^{t}$ & 9139.8 & 8047.5 & 8003 & 7815.5 & $\ldots$ & 9076.1 & 8628.9 & 8711.6 & 8697.7 & 8937 \\
\hline$B^{t}$ & 57.54 & 52.95 & 62.48 & 55.1 & $\ldots$ & 56.75 & 55.49 & 56.45 & 53.62 & 51.85 \\
\hline$C a^{t}$ & 42.27 & 49.23 & 44.46 & 44.33 & $\ldots$ & 65.23 & 70.85 & 80.46 & 68.62 & 69.98 \\
\hline$S a^{t}$ & 13.64 & 15.74 & 13.97 & 15.81 & $\ldots$ & 11.82 & 11.45 & 10.17 & 12.60 & 8.00 \\
\hline
\end{tabular}

The inclusion of precisely these control actions is dictated by common sense, their availability and the results of some selections for the best description. The estimation of the multiple correlation coefficient of the change in the dollar exchange rate $\Delta Y$ with all the controls $\Delta B, \Delta C a, \Delta M$, $\Delta S a, \Delta R$ (it is equal to the coefficient of determination $R^{2}$ in the regression analysis) was 0.6447 . The Wilks-Bartlett criterion rejects the hypothesis of pairwise independence of all these six values with a significance level of 0.0037 .

\section{Kalman Filter}

The Kalman filter is widely used in studies both in macroeconomics [4, 5, 6] and microeconomics [7].

Logarithm (1) to obtain a linear connection $s^{t}=r d^{t}-d^{t}$ of the proper value of the output quantity with the components of the state vector. The lower case letters denote the logarithms of the corresponding quantities. Taking logarithms (2) and (3), imply that $\ln (1+x) \approx x$ at small $\mathrm{x}$.

As shown by numerical experiments with these data, it is useful to introduce a "cobweb" cycle in the supply of rubles and dollars to the exchange market. As is known, the volume of the next delivery would necessarily be between the two previous ones. For this, in the logarithmic equations (2) and (3) in right hand parts replace $r d^{t} \rightarrow \frac{3}{4} r d^{t}+\frac{1}{4} r d^{t-1}$ and $d^{t} \rightarrow \frac{3}{4} d^{t}+\frac{1}{4} d^{t-1}$. Then, similarly to [8], we have a model of the system in the state space $x^{t+1}=F^{t} x^{t}+G^{t} u^{t}+w^{t}$, $y^{t}=H^{t} x^{t}+v^{t}$; where $w^{t}, v^{t}-$ noises of system and observation, $M w^{t}=0, M v^{t}=0, Q=M w^{t} w^{t T}, R=M v^{t} v^{t T}$.

State vector $x^{t}=\left(\begin{array}{lllll}r d^{t} & r d^{-1} & d^{t} & d^{t-1} \quad \Delta M^{t-1}\end{array}\right)^{T}$, control

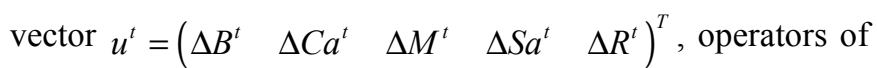
evolution and control $F^{t}=\left(\begin{array}{ccccc}3 / 4 & 1 / 4 & 0 & 0 & \frac{\beta_{0}}{R d^{t}} \\ 1 & 0 & 0 & 0 & 0 \\ 0 & 0 & 3 / 4 & 1 / 4 & 0 \\ 0 & 0 & 1 & 0 & 0 \\ 0 & 0 & 0 & 0 & 0\end{array}\right)$,
$G^{t}=\left(\begin{array}{ccccc}0 & 0 & \frac{\beta_{1}}{R d^{t}} & 0 & \frac{\beta_{2}}{R d^{t}} \\ 0 & 0 & 0 & 0 & 0 \\ \frac{\gamma_{0}}{D^{t}} & \frac{\gamma_{1}}{D^{t}} & \frac{\gamma_{2}}{D^{t}} & \frac{\gamma_{3}}{D^{t}} & 0 \\ 0 & 0 & 0 & 0 & 0 \\ 0 & 0 & 1 & 0 & 0\end{array}\right)$, observation operator $H=\left(\begin{array}{lllll}1 & 0 & -1 & 0 & 0\end{array}\right)$.

The estimation of the vector of model parameters $\theta=\left(\sigma_{v} \sigma_{w} \beta_{0} \beta_{1} \beta_{2} \gamma_{0} \gamma_{1} \gamma_{2} \gamma_{3}\right)^{T}$ was carried out by minimizing the mean square error of the Kalman filter (KF) prediction of the dollar rate $\operatorname{RMSE}(\theta)=\sqrt{\frac{1}{T u-(T l-1)} \sum_{t=T l}^{T u}\left[Y^{t+1}-\exp \left(\hat{y}^{t+1 \mid t}\right)\right]^{2}}$ $\hat{\theta}_{R M S E}=\underset{\hat{\theta}}{\arg \min } \operatorname{RMSE}(\hat{\theta})$.

Here $\hat{y}^{t+1 \mid t}$ is the forecast at time $t$ of the logarithm of the dollar rate at the next moment. $T l$ is the initial moment, $T u$ is the final moment of the training (fit) window.

Then, using the estimated parameters $\hat{\theta}_{R M S E}$, starting with the last state estimate, its covariance matrix and $u^{t}$, all at the moment $T u$, perform at moments $t$ from $T u+1$ to $T-1$ (getting $y^{t}$ and $u^{t}$ ) the Kalman filter forecasting for a month ahead.

The root-mean-square error of adequacy characterizes the average error of these forecasts $R M S E A=\sqrt{\frac{1}{T-2-T u} \sum_{t=T u+1}^{T-2}\left[Y^{t+1}-\exp \left(\hat{y}^{t+1 \mid t}\right)\right]^{2}} \quad$ (the forecast at time $T-1$ has no comparative; it is the goal of calculation). We choose the estimate of the initial state $\hat{x}^{0 \mid 0}=$ $\left(\begin{array}{lllll}9 & 9 & 5 & 5 & 0\end{array}\right)^{T}$, and the covariance matrix of this estimate equal to unit matrix $I . \quad R=\sigma_{v}^{2} I, Q=\sigma_{w}^{2} I$. Calculations are carried out in PTC Mathcad Prime ${ }^{\mathrm{TM}}$.

If to train (estimate) from $T l=0$ to $T u=20$, then the estimated parameters $\hat{\sigma}_{v}=24.129, \hat{\sigma}_{w}=0$, the positivity of the estimates $\hat{\beta}_{2}=1.762, \hat{\gamma}_{0}=2.126, \hat{\gamma}_{3}=1.734$ does not require comments, $\hat{\gamma}_{1}=1.473$ i.e. with the growth of foreign exchange reserves, the Central Bank, in a month, increases the sale of dollars, while with the decay it reduces it. This 
leads to severe fluctuations in the rate. $\hat{\beta}_{1}=33.246$ can mean fixation of profit in the stock market and its flow into the currency in a month, $\hat{\gamma}_{2}=0.439$ - the inflow of foreign investments a month after the growth of the MICEX index. The ratio of these coefficients is approximately equal to the dollar-ruble exchange rate. $\hat{\beta}_{0}=-8.718$ - what has grown in the stock market 2 months ago attracts rubles from the foreign exchange market.

You can roughly estimate the ratio of the ruble capacity of the dollar market and the ruble capacity of the MICEX index funds. If we take the effective $\hat{\beta}_{0}+\hat{\beta}_{1} \approx 20$, then the change in the MICEX index by 1 unit $(0.05 \%)$ causes a change $R d^{t}$ of 20 units of this calculation, which for absolute values roughly equal $\mathrm{e}^{10} \approx 20000$ means $0.1 \%$. Thus if we assume that exactly this money is transferred from the stock market to the foreign exchange market, then the capacity of the dollar market is half the capacity of the MICEX index funds.

The achieved RMSE $=1.93 \square$. To check the adequacy of the model remains 7 pairs of forecasts + the actual dollar rate (from 22 to 28). RMSEA, obviously, is not expected to be less than RMSE and was equal to $1.99 \square$. See Figure 1 .

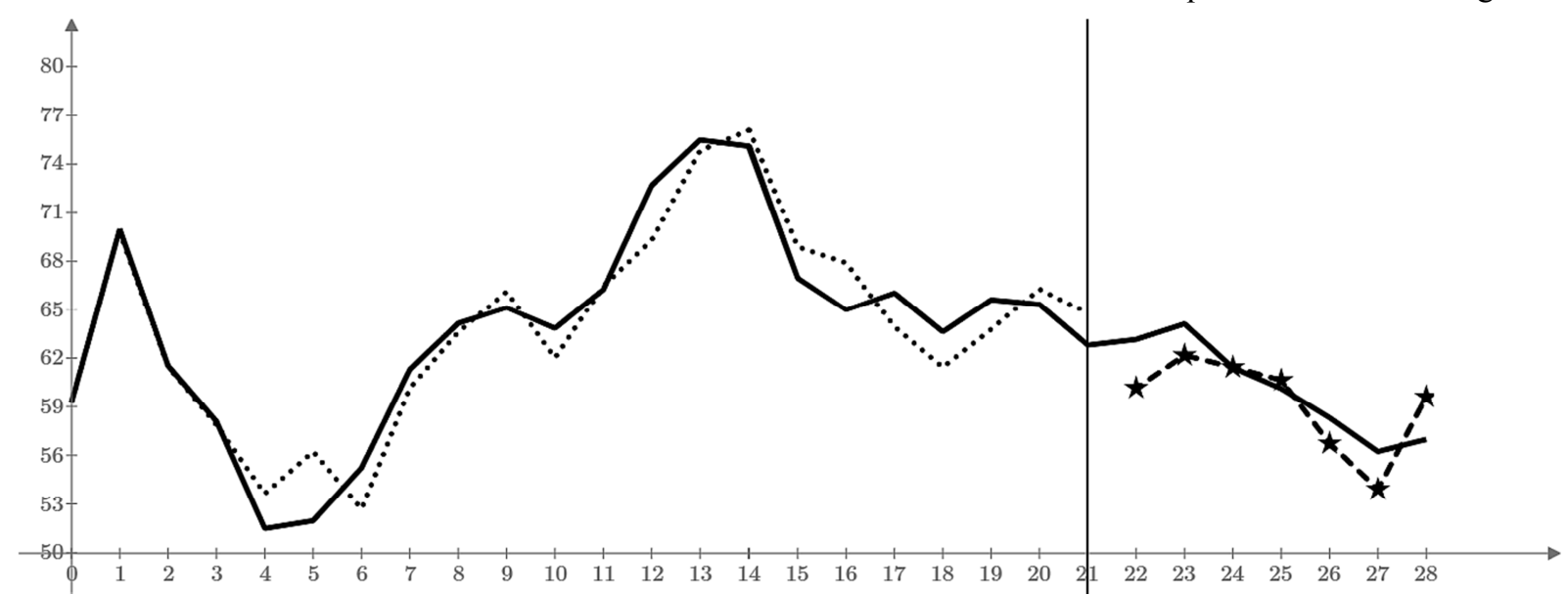

Figure 1. Monthly data from January 1, 2015 to May 1, 2017. The observed rate of USD / RUR (solid curve), adjusted to it the forecast for information a month ago (point curve - training), the forecast for the estimated model (dashed curve).

It can be seen that the dashed curve unduly reflects large fluctuations in the exchange rate, which were during the training period and are associated with specific causes for that time.

In practical forecasting, it is natural to become attached to the data of the most recent period, including the moment of forecasting. Therefore, perform training from $T l=16$ to $T u=$ $T-2=27$, i.e. take into account the last rate observation available $Y^{T-1=28}$. The results in Figure 2.

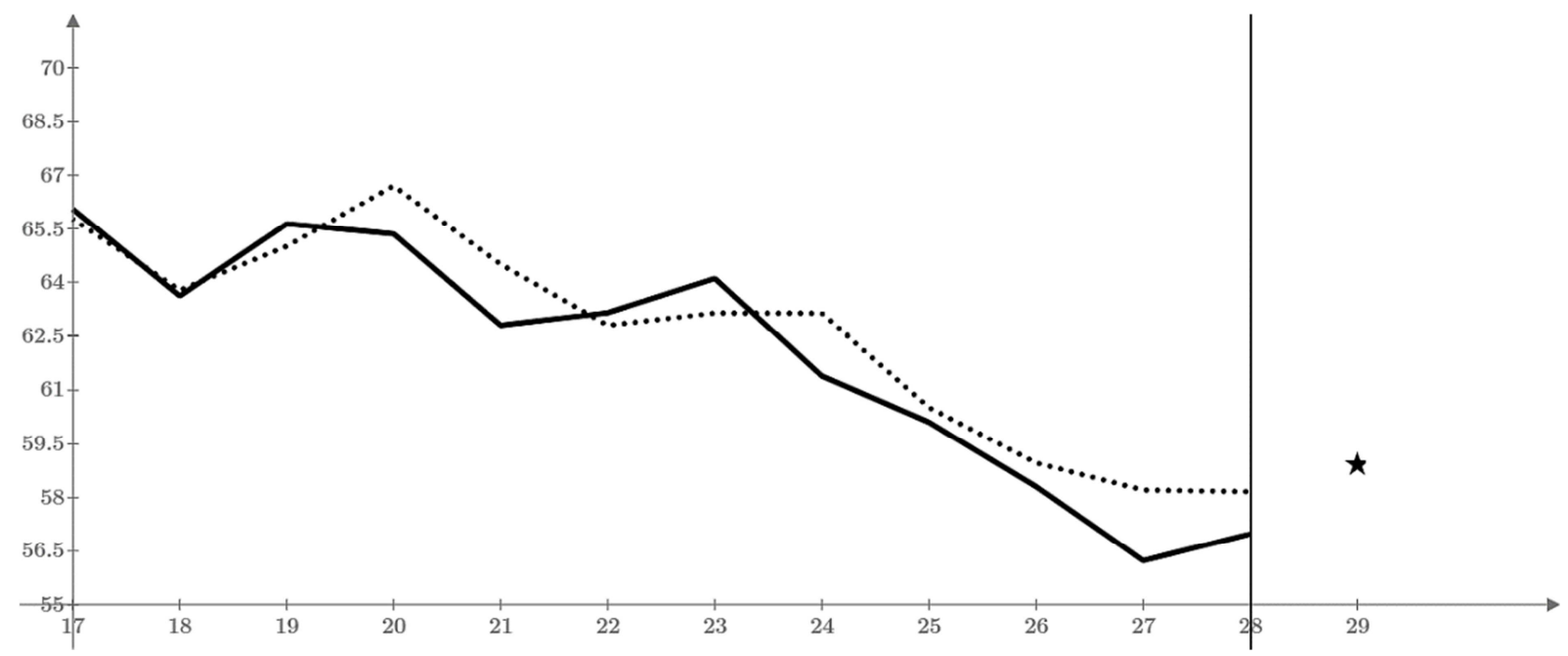

Figure 2. Monthly data from June 1, 2016 to May 1, 2017: the observed rate of USD / RUR (solid curve) and adjusted to it the forecast for information a month ago (point curve - training). The forecast for the estimated model for the future moment on June 1, 2017 (asterisk).

$\hat{\sigma}_{v}=0, \hat{\sigma}_{w}=13.108$, the estimate $\hat{\gamma}_{1}=-0.345$ changed sign reflects the efforts of the Central Bank to retain the course during this period. The achieved RMSE $=1.128$. The forecast for the estimated model for the future moment on
June 1, 2017 is 58.928.

For the same period of training, the behavior of the ruble and dollar masses participating in the exchange was studied. Estimates of these quantities are available through estimates 
of the states of the system. Trading volumes results on the MICEX (currency market) can confirm the correctness of these estimates. See Figure 3. The data is taken from the site http://www.moex.com.

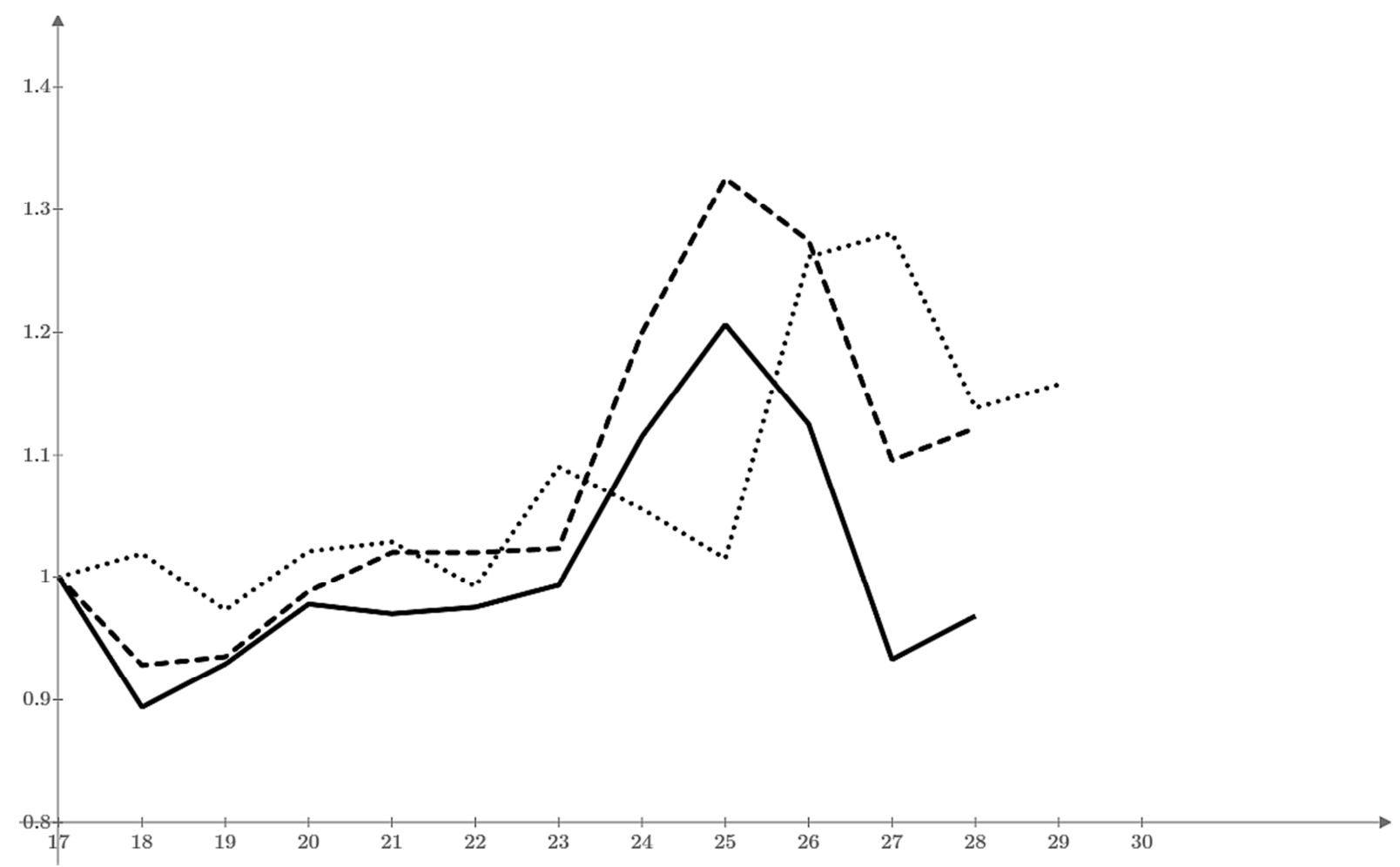

Figure 3. Monthly estimates from June 1, 2016 to May 1, 2017: ruble mass (solid curve), dollar mass (dashed curve). Half sum of the trading volumes on the MICEX (currency market) for the preceding and subsequent months (point curve). All values are normalized to their values on June 1, 2016.

It is interesting to compare the adequacy of forecasting for this model and the model proposed in [3]. If in that model (with the replacement of the time interval from day to month) we use the data of this work, then at $T l=0$ and $T u=20$ $\mathrm{RMSE}=4.36, \mathrm{RMSEA}=2.15$.

\section{Adaptive Kalman Filter}

Often the parameters of the system vary with time, so the task arises of their adaptive estimation and use. Note that sometimes this dynamics of the system parameters is a central (main) interest. In this paper, we propose the idea of a possible solution of this problem. The basic idea is similar to the EM method [9], when the phases of estimation and minimization are sequentially alternated. The algorithm is as follows.

1. The state estimate and its covariance matrix are assumed at time $t=0$, and also $w$ - the number of window points (width) of the primary estimation of the (vector) parameter $\theta$.

2. For $t=0, \ldots,(w-1) \mathrm{KF}$ estimation in the window is performed and compose $\operatorname{RMSE}(\hat{\theta})=\sqrt{\frac{1}{w} \sum_{t=0}^{w-1}\left[y^{t+1}-\hat{y}^{t+1 \mid t}(\hat{\theta})\right]^{2}} \quad, \quad$ where $y^{t}$ observed system output, $\hat{y}^{t+1 \mid t}$ - forecast on information at the time $t$, then, starting from a given initial parameter estimate $\hat{\theta}^{0}$, the parameter estimate $\hat{\theta}^{i}=\underset{\hat{\theta}}{\arg \min } \operatorname{RMSE}(\hat{\theta})$ is calculated and will be applied at any moment $0 \leq i \leq w$ of clause 3 .

3. The time $i$ is specified - the initial position of the beginning of the window of the regular parameter estimation and its width $v$. With value $\hat{\theta}^{i}$ we calculate $\hat{x}^{i \mid i}$ and $P^{i}$.

4. For every $t=i, \ldots,(T-v-1)$

a. the KF estimation in the window $t, \ldots, t+v-1$ is carried out and compose $\operatorname{RMSE}(\hat{\theta})=\sqrt{\frac{1}{w} \sum_{\tau=0}^{v-1}\left[y^{t+\tau+1}-\hat{y}^{t+\tau+1 \mid t+\tau}(\hat{\theta})\right]^{2}} \quad, \quad$ then starting with the $\hat{\theta}^{t}$ parameter estimation, the parameter estimate $\quad \theta^{\text {染 }}{ }^{1}=\underset{\hat{\theta}}{\arg \min } \operatorname{RMSE}(\theta)$ is calculated. With this value we calculate $\hat{x}^{t+1 \mid t+1}$ and $P^{t+1}$. These values will be applied for the next $t$ when moving the window 1 step forward.

b. at the point $t+v$, using the found with $\hat{\theta}^{t+1}$ state estimation $\hat{x}^{t+v \mid t+v}$ and control $u^{t+v}$, an adaptive forecast $\hat{y}^{t+v+1 \mid t+v}$ is made. To this point the resultant (by the immediately preceding window) parameter estimate $r \hat{\theta}^{t+v}=\hat{\theta}^{t+1}$ is assigned.

5. The dynamics of estimations of the parameter $r \hat{\theta}^{t+v}$, 
state $\hat{x}^{t+v \mid t+v}$ and forecast $\hat{y}^{t+v+1 \mid t+v}$ of the output quantity is collected from the moment $i+v$ up to the last moment of the available data $T-1$. Since the last one $y^{w}$ is used to estimate the parameters in the primary window, the final prediction $\hat{y}^{t+v+1 \mid t+v}$ must be considered as independent not earlier than at the moment $t+v \geq w$, and since $\min t=i$, then $i+v \geq w$.

Let us apply this adaptive KF to improve the RMSEA of dollar-ruble exchange rate in the range $t=22, \ldots, 28$. If we now choose $w=17, i=14, v=3$, then RMSEA is $1.39 \square$. This $v$ turned out to be the best, that is, the best predictions were obtained with the adjustment of the parameters from the data of the last 3 moments preceding the moment of forecasting. See Figure 4, which shows the observed data and forecasts on information at the time of the month before.

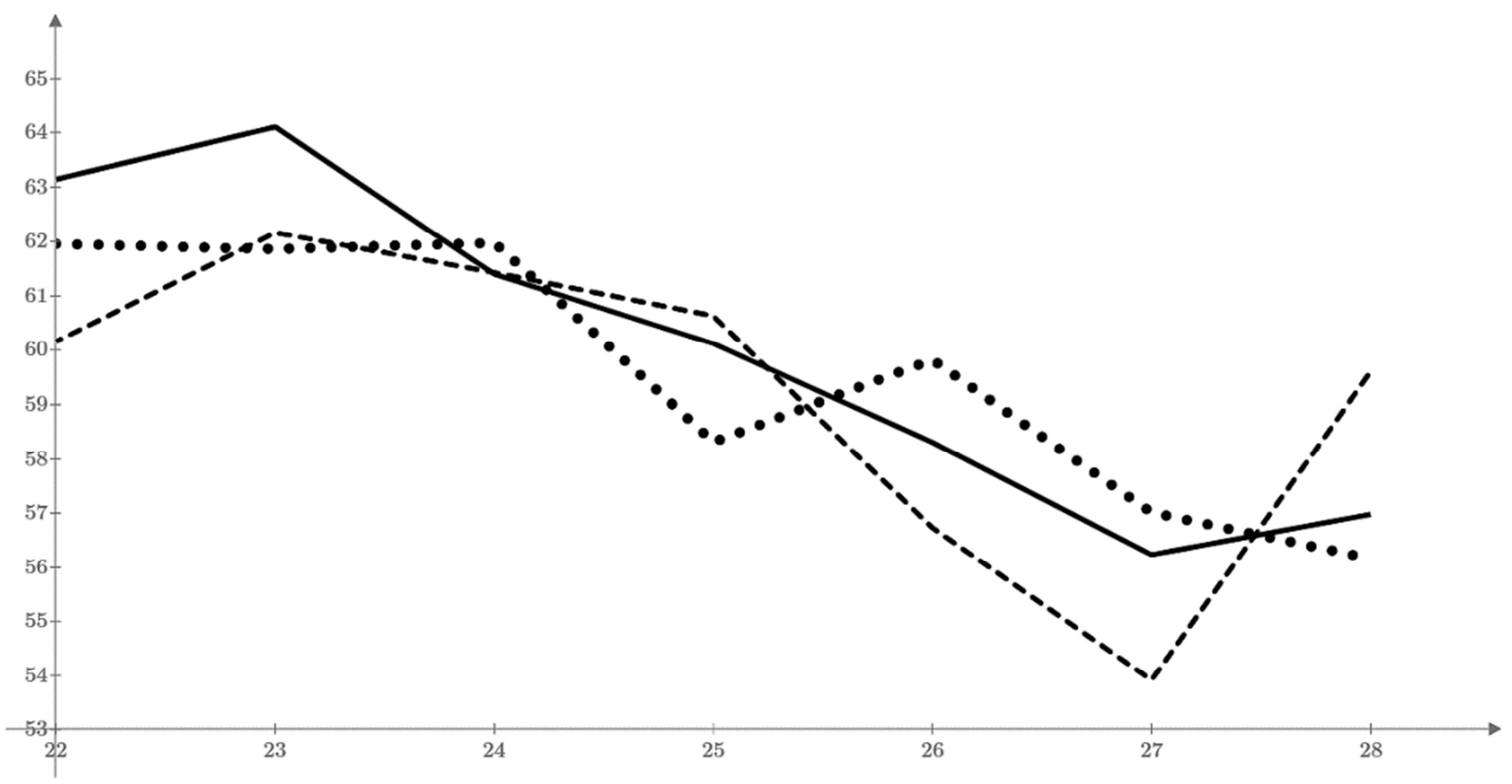

Figure 4. Monthly data from November 1, 2016 to May 1, 2017. Observed USD / RUR rate (solid curve), KF forecasts from Fig.1 (dashed curve), and forecasts for adaptive KF (point curve).

The dynamics of the estimated parameters of the model is shown in Figure 5. All estimates are normalized to their absolute values at $t=17: \hat{\beta}_{0}=-5.06, \hat{\beta}_{1}=6.90, \hat{\beta}_{2}=3.67, \hat{\gamma}_{0}=-0.33, \hat{\gamma}_{1}=0.77, \hat{\gamma}_{2}=0.21, \hat{\gamma}_{3}=3.02$.

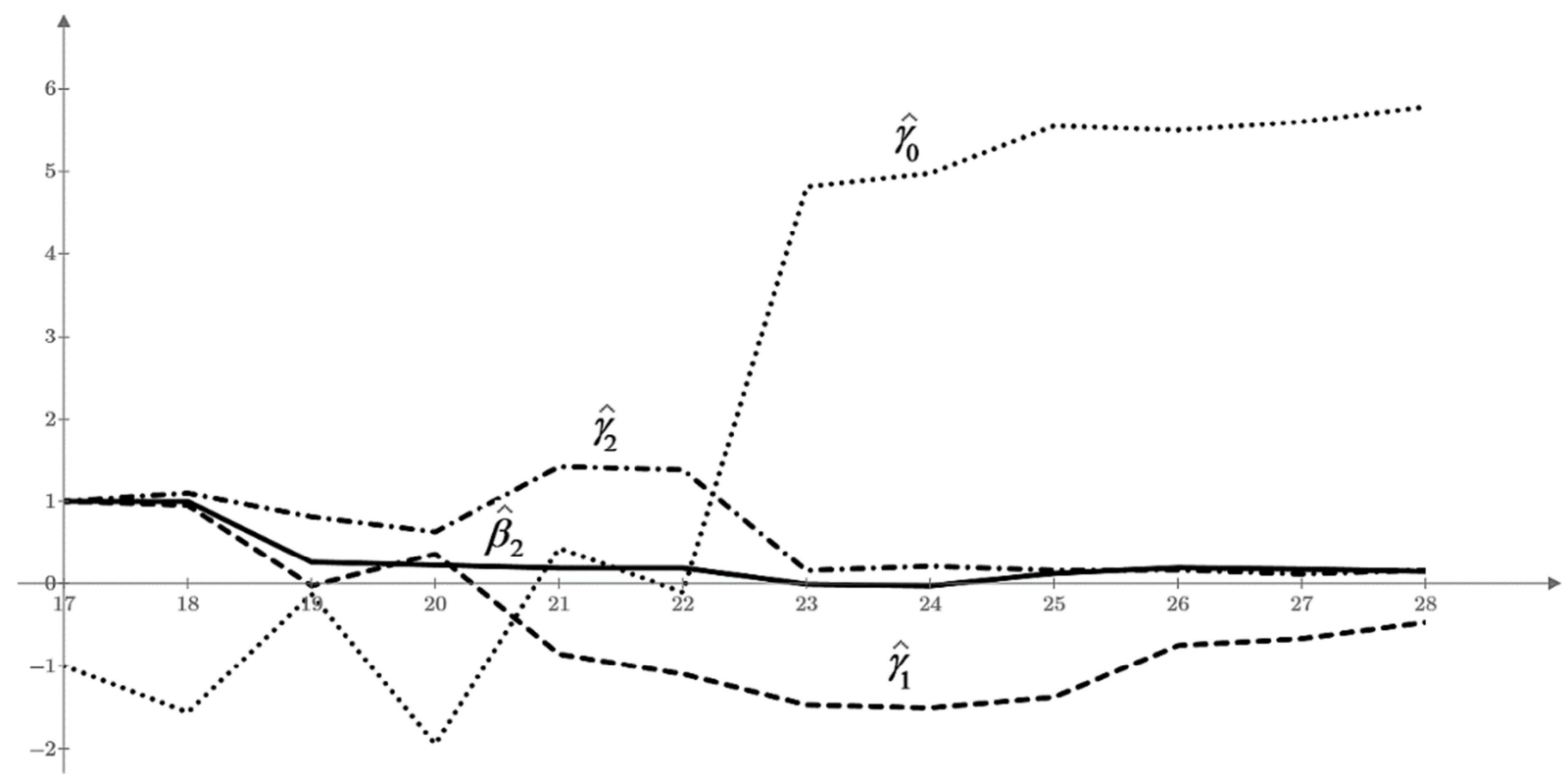

Figure 5. Monthly estimates of parameters from June 1, 2016 to May 1, 2017.

$\hat{\beta}_{2}$ - the change in the monetary base in recent months has slight effect on the investment of rubles in dollars. $\hat{\gamma}_{0}-$ in the 
summer of 2016, the increase in the price of oil reduced the inflow of dollars into the currency market (!), recently significantly increases. $\hat{\gamma}_{1}$ - shows the efforts of the Central Bank to retain the exchange rate, especially at the end of 2016 and early 2017. $\hat{\gamma}_{2}$ - the influx of dollar investments in Russian assets after the spike in October-November 2016 fell at large MICEX indices. Estimates $\hat{\beta}_{0}, \hat{\beta}_{1}$ and $\hat{\gamma}_{3}$ vary little.

The application of this adaptive KF to other economic problems can be found in [10].

\section{Conclusion}

A method is proposed for forecasting the exchange rate through the modeling of the monetary masses behavior of two respective currencies participating in the exchange.

Along with the use of conventional $\mathrm{KF}$, an adaptive extension of KF has been developed and applied, in which forecasts are made with model parameters optimized for the immediately preceding window. It gives more adequate (outof-sample) forecasts and reveals the dynamics of economic systems inner structure.

\section{References}

[1] Michele Ca'Zorzi, Marcin Kolasa and Michał Rubaszek, "Exchange rate forecasting with DSGE models," Journal of International Economics, vol. 107, pp. 127-146, 2017.

[2] Martin S. Eichenbaum, Benjamin K. Johannsen and Sergio Rebelo, "Monetary Policy and the Predictability of Nominal Exchange Rates," National Bureau of Economic Research Working Paper Series, No. 23158, 2017.
[3] S. M. Borodachev, "Prediction of the dollar to the ruble rate. A system-theoretic approach," AIP Conference Proceedings, vol. 1863 , p. 560025, 2017.

[4] M. Aguiar, S. Chatterjee, H. Cole and Z. Stangebye, "Quantitative Models of Sovereign Debt Crises," in Handbook of Macroeconomics, vol. 2, Amsterdam: North-Holland, 2016, pp 1697-1755.

[5] Martín Uribe and Stephanie Schmitt-Grohé, Open Economy Macroeconomics, Princeton University Press, 2017.

[6] S. M. Borodachev, "GDP and efficiency of Russian economy," AIP Conference Proceedings, vol. 1926, p. 020011, 2018.

[7] Yemei Qin, Hui Peng, Yanhui Xi, Wenbiao Xie, Yapeng Sun and Xiaohong Chen. "An adaptive modeling and asset allocation approach to financial markets based on discrete microstructure mode," Applied Soft Computing, vol. 43, pp. 390-405, 2016.

[8] S. M. Borodachev, "Unobservable characteristics of currency and stock markets system in Russia," paper presented in 4th International Multidisciplinary Scientific Conference on Social Sciences and Arts (SGEM 2017), Conference Proceedings Book 1, vol. 1, pp. 831-836.

[9] A. P. Dempster, N. M Laird, and D. B. Rubin, "Maximum Likelihood from Incomplete Data via the EM Algorithm," Journal of the Royal Statistical Society, Series B, vol. 39 (1), pp. 1-38, 1977.

[10] S. M. Borodachev, "Economic applications of Adaptive Kalman filter," paper presented in 27th International Scientific Conference on Economic and Social Development (Rome, 1-2 March 2018), Conference Proceedings Book, pp. 584-588. 